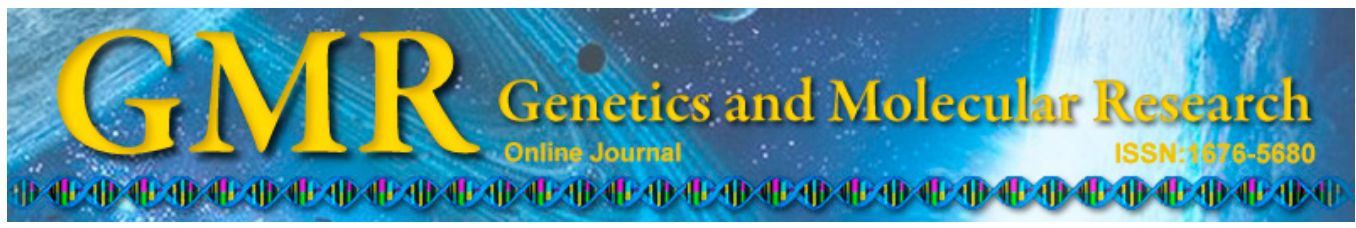

Short Communication

\title{
Development of novel microsatellite markers for conservation genetic studies of Vulpes vulpes (Canidae) by using next- generation sequencing method
}

\author{
J.-N. Yu', C.-U. Chung ${ }^{2}$, K.H. Oh ${ }^{1}$, B.-K. Lee ${ }^{3}$ and C.E. Lim ${ }^{1}$ \\ ${ }^{1}$ National Institute of Biological Resources, Environmental Research Complex, \\ Incheon, Korea \\ ${ }^{2}$ Species Restoration Technology Institute, Korea National Park Service, \\ Yeongju, Korea \\ ${ }^{3}$ Species Restoration Technology Institute, Korea National Park Service, \\ Gurye-gun, Korea \\ Corresponding author: C.E. Lim \\ E-mail: Chaelim@korea.kr
}

Genet. Mol. Res. 14 (2): 3980-3983 (2015)

Received May 28, 2014

Accepted November 11, 2014

Published April 27, 2015

DOI http://dx.doi.org/10.4238/2015.April.27.12

\begin{abstract}
The red fox, Vulpes vulpes (Canidae), is the most widely distributed terrestrial carnivore worldwide, but this species is classified as endangered in Korea. In this study, we developed 25 polymorphic microsatellite markers that included 3-13 (mean $=6.32$ ) alleles per locus using 22 red fox individuals. The most polymorphic locus was $F R(59) T G$ (13 alleles) and the least polymorphic loci were $F R(70) T G$ and $F R(182) A G$ (3 alleles each). No significant deviation from HardyWeinberg equilibrium $(\mathrm{P}<0.05)$ was observed for the 25 markers. Observed $\left(H_{\mathrm{O}}\right)$ and expected $\left(H_{\mathrm{E}}\right)$ heterozygosity varied from 0.182 to 1.000 and from 0.175 to 0.929 , respectively. These newly developed microsatellite markers will be useful for investigating the genetic diversity and population genetic structure of $V$. vulpes and will aid in
\end{abstract}


developing conservation strategies for this species.

Key words: Next-generation sequencing; Endangered species; Microsatellites; Red fox; Vulpes vulpes

\section{INTRODUCTION}

The red fox (Vulpes vulpes, Canidae) is a small carnivorous mammal that is widely distributed in Eurasia, northern Africa, and the Americas, and its status is generally stable across most of its range (IUCN, 2013). In South Korea, however, illegal killing to protect livestock or for fur, combined with habitat loss and fragmentation, have resulted in significant decline in the red fox population (Won and Smith, 1999). In the 1980s, the red fox was thought to be extinct and was listed as an "Endangered Species I" on the Korean Red List (Ministry of the Environment of Korea, 2005). With the exception of a dead red fox discovered in Yanggu, Gangwon Province in 2004, wild V. vulpes have not been observed in South Korea since the 1980s. A "Red Fox Restoration Project" was initiated in 2011 by the Ministry of the Environment of Korea. Recently, Yu et al. (2012) suggested that the introduction of individuals from genetically and geographically close northeastern populations, such as from North Korea, China, and Russia, would provide the best alternative for restoring $V$. vulpes in South Korea.

Here, we developed 25 polymorphic microsatellite markers to facilitate conservation and restoration of red fox in South Korea using next-generation sequencing (NGS). Twentytwo individuals of $V$. vulpes from North Korea and northeastern China were used to characterize these microsatellite markers.

\section{MATERIAL AND METHODS}

Total genomic DNA was isolated from blood, muscle tissue, or hair of the fox specimens using a DNeasy Blood \& Tissue Kit (Qiagen, Hilden, Germany), according to the manufacturer protocol. DNA quality was assessed by electrophoresis on a $1 \%$ agarose gel followed by evaluation using a spectrophotometer (Nanodrop, Technologies, Wilmington, DE, USA). To search for microsatellite fragments in $V$. vulpes, we used one DNA sample from Yanggu-gun, Gangwon Province in South Korea. Approximately 5-10 $\mu$ g genomic DNA was sequenced using one-quarter of a Roche GS-FLX-454 plate (454 Life Sciences, Branford, CT, USA) with a titanium platform, at the National Instrumentation Center for Environmental Management (NICEM, Seoul, South Korea).

The NGS data yielded 206,687 reads in FASTA format and a total of 71,115,299 base pairs (bp) from the genomic DNA of $V$. vulpes. After trimming off low-quality sequences, 174,425 reads remained $(65,562,274 \mathrm{bp})$. From these sequences, we chose 200 with higher copy numbers (c6) of di- and trinucleotide repeats for the amplification test and assessment of polymorphism. A primer set was designed for the flanking sequences of the microsatellite repeat motifs using Primer 3.0 (Rozen and Skaletsky, 2000). The optimal primer size was set to 18-26 bp, and an M13 sequence tag (M13-TGTAAAACGACGGCCAGT) was added to the 5'-end of the forward primer to allow fluorescent labeling during amplification (Schuelke, 2000).

PCR amplifications were performed on all 22 individuals in $20 \mu \mathrm{L}$ volumes containing 30-50 ng template DNA, $0.5 \mu \mathrm{L}$ dNTPs $(20 \mathrm{mM}), 1 \mu \mathrm{L}$ 10X PCR buffer containing 25 $\mathrm{mM} \mathrm{MgCl}$ (Takara, Japan), $0.25 \mu \mathrm{L}$ forward and $1 \mu \mathrm{L}$ reverse primers ( $8 \mathrm{pM}$ each), and 1 $\mu \mathrm{L}$ fluorescently labeled M13 primer (8 pM; labeled with 6-FAM, VIC, PET, and NED, Ap- 
plied Biosystems, Foster City, USA). The amplifications were conducted using the following cycling conditions: $5 \mathrm{~min}$ pre-denaturation at $94^{\circ} \mathrm{C}$ followed by 30 cycles of $30 \mathrm{~s}$ at $94^{\circ} \mathrm{C}, 45 \mathrm{~s}$ at $56^{\circ} \mathrm{C}$, and $45 \mathrm{~s}$ at $72^{\circ} \mathrm{C}$, followed by 8 cycles of $30 \mathrm{~s}$ at $94^{\circ} \mathrm{C}, 45 \mathrm{~s}$ at $53^{\circ} \mathrm{C}$, and $45 \mathrm{~s}$ at $72^{\circ} \mathrm{C}$, and then a final $20 \mathrm{~min}$ extension step at $72^{\circ} \mathrm{C}$.

The fluorescently labeled PCR products were genotyped on an ABI 3710XL DNA analyzer (Applied Biosystems, Foster City, CA, USA) using LIZ-500 as the internal size standard (Applied Biosystems, Foster City, CA, USA), and were visualized using the GeneMarker program (version 2.40, Softgenetics LLC). The data were checked for null alleles and scoring errors using Micro-Checker version 2.2.3 (van Oosterhout et al., 2004). Allele number per locus, observed $\left(H_{\mathrm{O}}\right)$ and expected $\left(H_{\mathrm{E}}\right)$ heterozygosity, and deviation from Hardy-Weinberg equilibrium $(H W E)$ were analyzed using Arlequin version 3.1 (Excoffier et al., 2007).

\section{RESULTS AND DISCUSSION}

A total of 200 microsatellite primers were screened in the $22 \mathrm{~V}$. vulpes individuals for amplification and polymorphism; 25 microsatellite markers were polymorphic, with the number of alleles per locus ranging from 3 to 13 (mean $=6.32$ ). The $H_{\mathrm{O}}$ and $H_{\mathrm{E}}$ values ranged from 0.182 to 1.000 and 0.175 to 0.929 , respectively (Tables 1 and 2). The remaining microsatellite markers were monomorphic or failed to amplify scorable products. No significant $(\mathrm{P}<0.05)$ deviations from $H W E$ were observed in locus-pair/populations in the 25 newly developed microsatellite markers. In addition, Micro-Checker analysis revealed no evidence for null alleles, scoring error, stuttering, or allelic dropout in any of the loci. Based on these results, we expect that the 25 newly developed microsatellite markers will be useful for estimating genetic diversity and will aid in genetic breeding, monitoring molecular ecology, and conserving genetic resources in $V$. vulpes.

Table 1. Information on the 25 microsatellite loci developed for Vulpes vulpes.

\begin{tabular}{|c|c|c|c|c|c|}
\hline Locus & $\begin{array}{l}\text { Repeat } \\
\text { motif }\end{array}$ & $\begin{array}{c}\text { Fluorescence } \\
\text { label }\end{array}$ & $\underline{\text { M13 tail }+F-p r i m e r ~}\left(5^{\prime} \rightarrow 3^{\prime}\right)$ & R-primer $\left(5^{\prime} \rightarrow 3^{\prime}\right)$ & $\begin{array}{c}\text { GenBank } \\
\text { accession No. }\end{array}$ \\
\hline $1 \mathrm{RF}(21) \mathrm{TG}$ & {$[\mathrm{TG}]_{\mathrm{n}}$} & 6-FAM & TGTAAAACGACGGCCAGTGGAGGCATTTTATTCCATCATA & TCGTGATCTCTCTTAAAGGTGC & KJ588476 \\
\hline $2 \mathrm{RF}(56) \mathrm{TG}$ & {$[\mathrm{TG}]_{\mathrm{n}}$} & PET & TGTAAAACGACGGCCAGTAATCAGGGGTCTTTTTCTCAAT & ACATCTGAAACCAAATGTTATATGTC & C KJ588477 \\
\hline $3 \mathrm{RF}(59) \mathrm{TG}$ & {$[\mathrm{TG}]_{\mathrm{n}}$} & VIC & TGTAAAACGACGGCCAGTGCTTGTTTTGAAGCTAGGAAGA & TCCCAAAGGAAAAGTAGACTCTC & KJ588478 \\
\hline $4 \mathrm{RF}(70) \mathrm{TG}$ & {$[\mathrm{TG}]_{\mathrm{n}}$} & NED & TGTAAAACGACGGCCAGTAAAAGAGGGCTACCCTCACTAC & CTGTGTGTCAGCTTATTTGGAA & KJ588479 \\
\hline $5 \mathrm{RF}(71) \mathrm{TG}$ & {$[\mathrm{TG}]_{\mathrm{n}}$} & VIC & TGTAAAACGACGGCCAGTTTCTAACTCAAAGGTGGCAAAT & TATTACTACCTCTCCCCTCCGT & KJ588480 \\
\hline $6 \mathrm{RF}(125) \mathrm{CA}$ & {$[\mathrm{CA}]_{\mathrm{n}}$} & 6-FAM & TGTAAAACGACGGCCAGTAGGCAAGGCTTTTAACAACATA & ACAAATTAAAACTTCCCCAGGT & KJ588481 \\
\hline $7 \mathrm{RF}(127) \mathrm{CA}$ & {$[\mathrm{CA}]_{\mathrm{n}}$} & VIC & TGTAAAACGACGGCCAGTACATCAAAGCTACCAACAGAGG & ACAAGAAACAGAATCATGGGAC & KJ588482 \\
\hline $8 \mathrm{RF}(131) \mathrm{CA}$ & {$[\mathrm{CA}]_{\mathrm{n}}$} & VIC & TGTAAAACGACGGCCAGTCCAGGTATTTACCCAAGTGAAA & GCAGGAATACTCCATTGTTGTT & KJ588483 \\
\hline $9 \mathrm{RF}(136) \mathrm{CA}$ & {$[\mathrm{CA}]_{n}$} & PET & TGTAAAACGACGGCCAGTTAATTCAGGGCATGGTTTACTC & CCAGGGATACCTGAAGTTGTAA & KJ588484 \\
\hline $10 \mathrm{RF}(139) \mathrm{CA}$ & {$[\mathrm{CA}]_{\mathrm{n}}$} & VIC & TGTAAAACGACGGCCAGTTAATTATTTGGTAGGCAGGGTG & TGAATTGTGAATGATAGCTTGC & KJ588485 \\
\hline $11 \mathrm{RF}(143) \mathrm{CA}$ & {$[\mathrm{CA}]_{\mathrm{n}}$} & VIC & TGTAAAACGACGGCCAGTTGGTGTACATCTCTGCTTCACT & CATTTACCAGTCATTGGATGTG & KJ588486 \\
\hline $12 \mathrm{RF}(147) \mathrm{CA}$ & {$[\mathrm{CA}]_{\mathrm{n}}$} & VIC & TGTAAAACGACGGCCAGTGCAATCATGTGTGTTGAATAGG & TTCCCTACGTACTGCTTTTGTT & KJ588487 \\
\hline $13 \mathrm{RF}(155) \mathrm{CA}$ & {$[\mathrm{CA}]_{\mathrm{n}}$} & VIC & TGTAAAACGACGGCCAGTATGTTTTTCTTCCAGTGCTTGT & CTTCACTTCCAGAATACCTCCA & KJ588488 \\
\hline $14 \mathrm{RF}(156) \mathrm{CA}$ & {$[\mathrm{CA}]_{\mathrm{n}}$} & PET & TGTAAAACGACGGCCAGTTTCCCTAGAAGAACGTGACCTA & GATTTCCTCACCTTGTGAACAT & KJ588489 \\
\hline $15 \mathrm{RF}(157) \mathrm{CA}$ & {$[\mathrm{CA}]_{\mathrm{n}}$} & 6-FAM & TGTAAAACGACGGCCAGTCAATTGGCTTATCCTTCAAAAC & CTTCCTTAAGTCTCCCATTCCT & KJ588490 \\
\hline $16 \mathrm{RF}(162) \mathrm{ATC}$ & {$[\mathrm{ATC}]_{\mathrm{n}}$} & NED & TGTAAAACGACGGCCAGTTACCATTACGTTAGGGATTTGG & CCTCTGTGTTCATTCCAGAAGT & KJ588491 \\
\hline 17 RF(163)ATC & {$[\mathrm{ATC}]_{\mathrm{n}}$} & VIC & TGTAAAACGACGGCCAGTCTTCCTTCCCTCATTTCTTTCT & TGTATGCCATATAGTAGGTGCTTT & KJ588492 \\
\hline $18 \mathrm{RF}(165) \mathrm{TGA}$ & {$[\mathrm{TGA}]_{\mathrm{n}}$} & 6-FAM & $\underline{\text { TGTAAAACGACGGCCAGTGGCTTTGTAAGGAAGAGCATAA }}$ & CGCTGCTCTCAGAATAGAGAAC & KJ588493 \\
\hline $19 \mathrm{RF}(174) \mathrm{GA}$ & {$[\mathrm{GA}]_{\mathrm{n}}$} & NED & TGTAAAACGACGGCCAGTATCAATGAAAGTGAGAGCTGGT & TTTTAGGTTGTTCTATTTCTTGCC & KJ588494 \\
\hline $20 \mathrm{RF}(176) \mathrm{AG}$ & {$[\mathrm{AG}]_{\mathrm{n}}$} & PET & TGTAAAACGACGGCCAGTTTTGATCTGCACTTTTTCAGTG & TGGCGTCAATAGTTTAAGTGTG & KJ588495 \\
\hline $21 \mathrm{RF}(182) \mathrm{AG}$ & {$[\mathrm{AG}]_{\mathrm{n}}$} & NED & TGTAAAACGACGGCCAGTTCAAGTTATGGATGGACACAGA & TTAGAATTCAGATAGCCCCTGA & KJ588496 \\
\hline $22 \mathrm{RF}(195) \mathrm{AAT}$ & {$[\mathrm{AAT}]_{\mathrm{n}}$} & VIC & TGTAAAACGACGGCCAGTACTTGCAGGTGCCCTTATG & TGTTTGTGCTTACTGTCAGAGG & KJ588497 \\
\hline $23 \mathrm{RF}(198) \mathrm{AAT}$ & {$[\mathrm{AAT}]_{\mathrm{n}}$} & NED & TGTAAAACGACGGCCAGTGCCCACACTCTGATCTGTATCT & AAAGGTTTGAGTTTGAAGGGTT & KJ588498 \\
\hline $24 \mathrm{RF}(199) \mathrm{AAT}$ & {$[\mathrm{AAT}]_{\mathrm{n}}$} & VIC & TGTAAAACGACGGCCAGTTGCTTGATTTCAACTTAGGTCA & AACATGAGCTTAAACTCCCAAC & KJ588499 \\
\hline $25 \mathrm{RF}(200) \mathrm{AG}$ & {$[\mathrm{AG}]_{\mathrm{n}}$} & PET & TGTAAAACGACGGCCAGTCTCTCTCAACCTTTCTCCTGAA & TCTGCTTCCACTCAGATCATAA & KJ588500 \\
\hline
\end{tabular}


Table 2. Characterization of 25 microsatellite loci for Vulpes vulpes.

\begin{tabular}{|c|c|c|c|c|c|c|}
\hline Locus & No. of individuals & $N_{\mathrm{A}}$ & Allele size (allelic range) & $H_{\mathrm{O}}$ & $H_{\mathrm{E}}$ & pHWE \\
\hline $\mathrm{RF}(21) \mathrm{TG}$ & 22 & 6 & $228-241(13)$ & 0.818 & 0.816 & 0.800 \\
\hline $\mathrm{RF}(56) \mathrm{TG}$ & 22 & 5 & $281-289(8)$ & 0.727 & 0.709 & 0.846 \\
\hline RF(59)TG & 22 & 13 & $257-287(30)$ & 0.909 & 0.929 & 0.779 \\
\hline $\mathrm{RF}(70) \mathrm{TG}$ & 22 & 3 & $263-269(6)$ & 0.409 & 0.415 & 0.460 \\
\hline $\mathrm{RF}(71) \mathrm{TG}$ & 22 & 5 & $345-325(10)$ & 0.682 & 0.708 & 0.497 \\
\hline $\mathrm{RF}(125) \mathrm{CA}$ & 22 & 6 & $151-161(10)$ & 0.500 & 0.569 & 0.435 \\
\hline $\mathrm{RF}(127) \mathrm{CA}$ & 22 & 7 & $214-226(12)$ & 0.864 & 0.809 & 0.684 \\
\hline $\mathrm{RF}(131) \mathrm{CA}$ & 22 & 8 & $188-203(15)$ & 0.545 & 0.555 & 0.296 \\
\hline $\mathrm{RF}(136) \mathrm{CA}$ & 22 & 4 & $238-244(6)$ & 0.682 & 0.597 & 1.000 \\
\hline $\mathrm{RF}(139) \mathrm{CA}$ & 22 & 4 & $222-234(12)$ & 0.318 & 0.289 & 1.000 \\
\hline $\mathrm{RF}(143) \mathrm{CA}$ & 22 & 7 & $230-242(12)$ & 0.591 & 0.651 & 0.096 \\
\hline $\mathrm{RF}(147) \mathrm{CA}$ & 22 & 4 & $285-293(8)$ & 0.409 & 0.453 & 0.470 \\
\hline $\mathrm{RF}(155) \mathrm{CA}$ & 22 & 11 & $279-309(30)$ & 0.864 & 0.882 & 0.627 \\
\hline $\mathrm{RF}(156) \mathrm{CA}$ & 22 & 10 & $322-342(20)$ & 0.818 & 0.871 & 0.247 \\
\hline $\mathrm{RF}(157) \mathrm{CA}$ & 22 & 12 & $280-322(42)$ & 0.864 & 0.863 & 0.974 \\
\hline RF(162)ATC & 22 & 6 & $217-229(12)$ & 1.000 & 0.769 & 0.215 \\
\hline RF(163)ATC & 22 & 5 & $260-271(11)$ & 0.364 & 0.461 & 0.061 \\
\hline RF(165)TGA & 22 & 6 & $278-292(14)$ & 0.682 & 0.696 & 0.592 \\
\hline RF(174)GA & 22 & 5 & $219-244(25)$ & 0.182 & 0.175 & 1.000 \\
\hline $\mathrm{RF}(176) \mathrm{AG}$ & 22 & 5 & $241-249(8)$ & 0.500 & 0.564 & 0.278 \\
\hline $\mathrm{RF}(182) \mathrm{AG}$ & 22 & 3 & $231-235$ (4) & 0.227 & 0.280 & 0.431 \\
\hline RF(195)AAT & 22 & 5 & $253-268(15)$ & 0.636 & 0.628 & 0.815 \\
\hline RF(198)AAT & 22 & 5 & $254-266(12)$ & 0.636 & 0.773 & 0.487 \\
\hline RF(199)AAT & 22 & 7 & $272-289(15)$ & 0.727 & 0.687 & 0.353 \\
\hline $\mathrm{RF}(200) \mathrm{AG}$ & 22 & 6 & $306-320(14)$ & 0.727 & 0.736 & 0.777 \\
\hline
\end{tabular}

$N_{\mathrm{A}}=$ number of alleles; $H_{\mathrm{O}}=$ observed heterozygosity; $H_{\mathrm{E}}=$ expected heterozygosity; $p H W E=\mathrm{P}$ value for HardyWeinberg equilibrium tests for each marker. The size ranges include 18 base pairs of the M13 tail.

\section{ACKNOWLEDGMENTS}

The authors thank the anonymous reviewers for providing valuable comments on the manuscript. Research supported by the Post-Doctoral Fellowships Program "The Genetic Evaluation of Important Biological Resources" from the National Institute of Biological Resources, Korea.

\section{REFERENCES}

Excoffier L, Laval G and Schneider S (2007). Arlequin (version 3.0): an integrated software package for population genetics data analysis. Evol. Bioinform. 1: 47-50.

IUCN (2013). IUCN Red List of Threatened Species. Version 2013.2. Available at [www.iucnredlist.org]. Accessed May $28,2014$.

Ministry of the Environment of Korea (2005). Government Notification 2005-20. Gwancheon-shi, Gyeongggi-do, Korea.

Rozen S and Skaletsky H (2000). Primer3 on the WWW for general users and for biologist programmers. In: Bioinformatics methods and protocols (Misener S and Krawetz SA, eds.). Humana Press, New Jersey, 365-386.

Schuelke M (2000). An economic method for the fluorescent labeling of PCR fragments. Nat. Biotechnol. 18: 233-234.

van Oosterhout C, Hutchinson WF, Wills DPM and Shipley P (2004). MICRO-CHECKER: software for identifying and correcting genotyping errors in microsatellite data. Mol. Ecol. Notes 4: 535-538.

Won C and Smith KG (1999). History and current status of mammals of the Korean peninsula. Mammal Rev. 29: 3-36.

Yu JN, Han SH, Kim BH, Kryukov AP, et al. (2012). Insights into Korean red fox (Vulpes vulpes) based on mitochondrial cytochrome b sequence variation in East Asia. Zool. Sci. 29: 753-760. 